\title{
Metodologia de avaliação metrológica da medição de energia para analisadores de desfibriladores e cardioversores
}

\author{
Conrado Almeida de Oliveira, José Carlos Teixeira de Barros Moraes*
}

Resumo Neste artigo descreve-se uma pesquisa na qual se desenvolveu um analisador de desfibriladores e cardioversores e se executou sua avaliação metrológica, sendo apresentados os resultados obtidos. A avaliação das incertezas de medição do analisador foi realizada de acordo com as determinações da versão brasileira do EA-4/02 - Expressão da Incerteza de Medição de Calibração publicado pelo INMETRO. Os resultados obtidos mostraram-se adequados às exigências de exatidão determinadas pelas normas ANSI/AAMI DF2 (American..., 1996) e ANSI/AAMI DF80 (American..., 2003), tendo apresentado uma incerteza expandida relativa para as medições de energia igual a $1,0 \%$ no pior caso. Nesta pesquisa foi também realizada uma análise da incerteza definicional do processo de calibração utilizado e avaliou-se a capacidade do processo. Para os cálculos da capacidade do processo, foi determinado um erro máximo para as medições de energia igual a $5 \%$ ou $1,0 \mathrm{~J}$, o que for maior. Este erro máximo é igual a 1/3 do erro máximo admissível para a energia entregue pelos desfibriladores, em outras palavras, é igual ao erro máximo admissível para um instrumento de medição pela Norma ANSI/AAMI DF2 (American..., 1996). Os resultados obtidos para a capacidade do procedimento de calibração mostraram-se adequados para as especificações de um analisador de desfibriladores e cardioversores, tendo obtido como resultado, no pior caso, um índice de capacidade $C_{m}$ igual a 2,87, que é bem superior, portanto, a 1,0, sendo este o limite mínimo para que o procedimento seja considerado capaz de calibrar a medição de energia sem atribuir uma incerteza definicional demasiadamente grande.

Palavras-chave Avaliação, Metrologia, Analisador, Desfibrilador, Cardioversor.

\section{Methodology for metrological evaluation of power measurement for defibrillators and cardioverters analyzers}

\footnotetext{
Abstract This paper describes a research involving the development of a defibrillator and cardioversor analyzer and its metrological evaluation. The evaluation results are presented. The evaluation of measurement uncertainties was performed according to the directives of the Brazilian version of the EA-4/02 - Expressing Uncertainty in Calibration Measurements - published by INMETRO. The results conformed to the accuracy prescriptions defined in the ANSI/AAMI DF2 (American..., 1996) and ANSI/AAMI DF80 (American..., 2003) standards, presenting an expanded relative uncertainty of energy measurements of $1.0 \%$ in worst cases. An uncertainty analysis was performed for the calibration process employed in the research and the process capacity was evaluated. A maximum error of $5 \%$ or $1.0 \mathrm{~J}$, whichever is greater, was determined for the energy measurements taken to calculate process capacity. The maximum error is 1/3 of the maximum error acceptable for the energy delivered by the defibrillators, i.e., it is the maximum error acceptable for a measurement device according to the ANSI/AAMI DF2 (American..., 1996) standard. The results for the capacity of the calibration procedure were shown to be appropriate for the specifications of a defibrillator and cardioversor analyzer. At worst case the capacity index $C_{m}$ was 2.87, well above the threshold of 1.0, which is the minimum value needed to consider the procedure as capable of calibrating energy measurements without incurring in a large definition uncertainty.
}

Keywords Evaluation, Metrology, Analyzer, Defibrillators, Cardioverters. 


\section{Extended Abstract}

\section{Introduction}

The safe and effective operation of defibrillators and cardioverters require preventive and corrective maintenance. It is necessary, therefore, to conduct frequent evaluations of equipment performance. This evaluation is normally performed by a defibrillator and cardioversor analyzer.

Defibrillators and cardioverters require careful and responsible analysis of their multiple specifications. A complete and tried description of the minimum requirements for defibrillator and cardioverter analyzers is presented in Oliveira (2010). An important specification is the output energy value for defibrillators and cardioverters. This is the parameter adopted in this work to exemplify necessary procedures methodologically accepted for the definition and evaluation of defibrillator and cardioverter analyzers. Other characteristics should be considered and evaluated in similar manner

This article presents a developed defibrillator and cardioverter analyzer and a methodology, which can be used as a guide by manufacturers and users, to analyze its characteristics.

\section{Materials and Methods}

\section{General description of the circuit}

Figure 1 shows the block diagram for the defibrillator and cardioverter analyzer developed in this study. It is easy to use and capable of measuring the defibrillatory pulse delivered to a non-inductive resistance of $50 \Omega \pm 2.0 \%(<10 \mu \mathrm{H})$.

The defibrillatory discharge is applied through the application discs of the analyzer with which the user should couple the paddles of the tested defibrillator. The impedance between discs simulates the trans-thoracic impedance of a patient. The discharged defibrillatory pulse is attenuated and filtered before measuring by the $A / D$ converter. The $A / D$ converter is linked to the mainboard, transmitting the measurement results. The measurement results are then processed and the energy and other defibrillatory pulse parameters are determined.

\section{Procedure for calibration of energy measurements}

To calibrate energy measurements, the uncertainty in measurement was determined according to the prescriptions of the Brazilian version of the EA-4/02 (Instituto..., 1999) and the JCGM 100:2008 (Bureau..., 2008). The procedure followed the one indicated in item 7 , step by step procedure to calculate uncertainty in measurement, in the Brazilian version of EA-4/02, Expression of the Uncertainty of Measurement in Calibration (Instituto..., 1999).
The first step in this procedure is the modeling of the measurement. The mathematical model for the energy measurements of the developed defibrillator analyzer is presented in Equation 2. The second step in this procedure relates all uncertainty sources through an uncertainty analysis.

The third step derives from Equation 2 and is based on the calculation methods for the measurement uncertainty defined in EA-4/02 (Instituto..., 1999) and JCGM 100:2008 (Bureau..., 2008). Equation 3 is defined for the relative combined variance of the energies determined by measurements performed in the $\mathrm{CH} j$ channel of the A/D converter, where $\mathrm{CH} j$ is equal to $\mathrm{CHO}$ or $\mathrm{CH} 1$.

The parts of Equation 3 are determined in the fourth step of this procedure. The relationship between the calculated value of the effective voltage of a periodic signal and the energy value of the signal was used to calculate the relative combined variance of $\int V_{\mathrm{CH} 0}^{2}(t) d t$ and $\int V_{\mathrm{CH} 1}^{2}(t) \cdot d t$.

The fifth step in this procedure derives from Equation 3 and the results yielded in the fourth step. The relative combined variance is calculated for the energies determined by measurements performed in the $\mathrm{CH} j$ channel of the A/D converter. The relative uncertainty of the energies determined by their respective relative variances is also calculated in this fifth step.

The sixth step in this procedure is the determination of the expanded relative uncertainties for a coverage probability of $95.45 \%$. The relative definition uncertainty obtained in the calibration process for performing energy measurements with the analyzer is calculated using Equation 20.

\section{Results}

Tables 2-9 present the results obtained with the proposed procedure for uncertainty in measurement.

\section{Discussion and Conclusion}

The calibration of energy measurements performed in the developed analyzer showed that it presents expanded relative uncertainty of energy measurements of $1.0 \%$ in worst cases, as shown in Table 8. Those results are appropriate for a defibrillator and cardioverter analyzer.

A capacity evaluation of the methodology applied in the calibration was performed. The evaluation considered the maximum acceptable error for energy measurements performed with the defibrillator and cardioversor analyzer, which is $\pm 5 \%$ or $\pm 1.0 \mathrm{~J}$, whichever is higher.

Table 9 shows that the proposed calibration procedure presented an uncertainty of 0.57 and $0.58 \%$ for measurements performed with channels $\mathrm{CHO}$ and $\mathrm{CH} 1$ of the $\mathrm{A} / \mathrm{D}$ converter, respectively, and a capacity coefficient $C_{m}$ of 2.92 and 2.87 , respectively. 


\section{Introdução}

A avaliação periódica de desfibriladores e cardioversores durante todo o seu ciclo de vida é essencial para garantir o seu funcionamento eficaz e seguro. As características metrológicas desses equipamentos devem ser rigorosamente avaliadas. Assim, este artigo apresenta uma metodologia para análise destas características para ser utilizada como guia para fabricantes e usuários.

Este trabalho apresenta um analisador de desfibriladores e cardioversores desenvolvido de acordo com as exigências estabelecidas por normas técnicas internacionais e nacionais (Associação..., 1997), considerando as especificações fundamentais exigidas para esta classe de analisadores (Oliveira, 2010). O analisador é apresentado em diagrama de blocos com a descrição de seu funcionamento básico. Para a sua avaliação, foram compiladas especificações necessárias para analisadores de desfibriladores e cardioversores (Oliveira, 2010). Dentre estas especificações, sobressai-se a medição da energia de saída dos desfibriladores e cardioversores.

Neste artigo apresenta-se o procedimento completo e detalhado para a calibração das medições de energia aplicado ao equipamento desenvolvido para exemplificar o método metrologicamente aceitável e necessário para a definição e avaliação das especificações de analisadores de desfibriladores e cardioversores (Instituto..., 2009). As outras especificações devem ser consideradas e avaliadas de maneira análoga.
Os resultados são apresentados, analisados e discutidos. Uma metodologia e avaliação metrológica de outros parâmetros do analisador de desfibriladores e cardioversores podem ser encontradas em Oliveira (2010).

\section{Materiais e Métodos}

\section{$O$ analisador de desfibriladores $e$ cardioversores ensaiado}

$\mathrm{O}$ analisador de desfibriladores e cardioversores desenvolvido é de fácil utilização e capaz de medir a energia do pulso desfibrilatório entregue por um desfibrilador.

Na Figura 1 é apresentado o diagrama em blocos do analisador de desfibriladores e cardioversores desenvolvido nesta pesquisa.

A descarga desfibrilatória é aplicada nos discos do analisador sobre uma resistência de $50 \Omega \pm 2,0 \%$ não indutiva $(<10 \mu \mathrm{H})$, que simula a impedância transtorácica de um paciente. O pulso desfibrilatório passa pelo bloco atenuador e segue pelo bloco filtro de entrada para então ser aquisitado pelo bloco $\mathrm{A} / \mathrm{D}$, conforme mostra a Figura 1.

O bloco atenuador é o responsável por condicionar o sinal do pulso de descarga para valores adequados para a medição e é constituído por um divisor resistivo. O bloco filtro de entrada é constituído por um circuito amplificador de instrumentação e um filtro tipo Butterworth de $2^{\circ}$ ordem com realimentação múltipla e com frequência de corte $f_{C}=5 \mathrm{kHz}$.

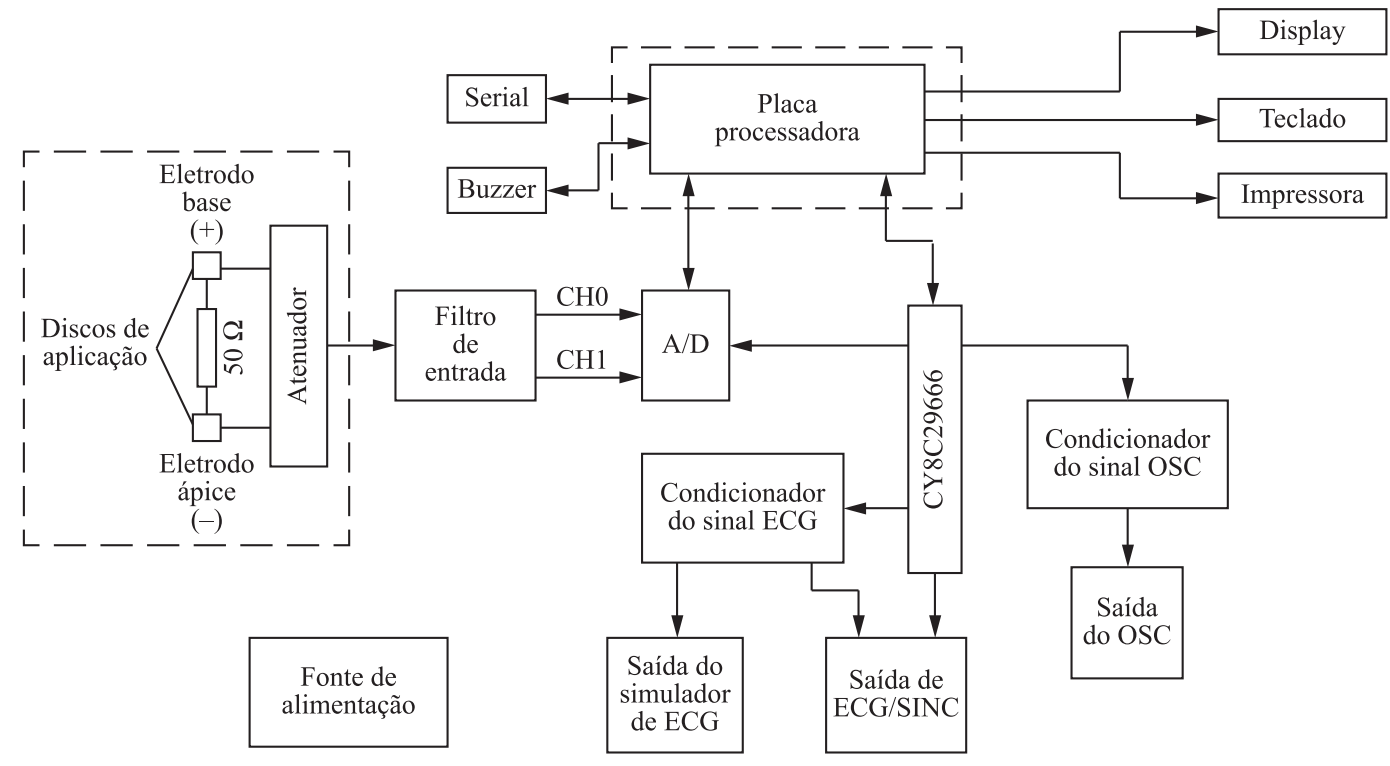

Figura 1. Diagrama em blocos do analisador de desfibrilador.

Figure 1. Block diagram of the defibrillator analyzer. 
O bloco A/D é constituído por um conversor analógico digital (National Semiconductor, ADC12048) com resolução de 12 bits mais um bit de sinal, e 8 entradas diferenciais analógicas multiplexáveis. A taxa de amostragem do sinal aquisitado é de $10 \mathrm{kHz}$.

$\mathrm{O}$ analisador realiza medições de até $1.000 \mathrm{~J}$ de energia com resolução de $0,01 \mathrm{~J}$ em uma janela de medição de $24 \mathrm{~ms}$. A tensão mínima de pico para que o analisador detecte a aplicação de pulso de descarga é de $75 \mathrm{~V}$. Realiza também as medições do tempo de carga do desfibrilador, do tempo de sincronismo de descargas sincronizadas, da tensão (faixa de $0 \mathrm{a} 6 \mathrm{kV}$ ) e da corrente de pico (faixa de 0 a $120 \mathrm{~A}$ ) do pulso desfibrilatório.

A forma de onda do pulso desfibrilatório é apresentada em um display tipo LCD de $256 \times 64$ pixels e em uma saída para osciloscópio. Todos os parâmetros aferidos podem ser transmitidos a um computador por meio de uma interface serial RS-232. A escala de tempo da forma de onda do pulso desfibrilatório apresentada no display é fixa e igual a $0,1 \mathrm{~ms} /$ pixel. Já a escala de amplitude é variável para facilitar a sua visualização.

$\mathrm{O}$ analisador contém um gerador de sinais de ECG utilizado para medir o tempo decorrido entre a detecção da onda $\mathrm{R}$ pelo cardioversor e a aplicação do pulso desfibrilatório (tempo de sincronismo). O gerador de sinais de ECG também pode reproduzir formas de onda de arritmias (passíveis ou não de desfibrilação) para avaliar a capacidade de desfibriladores externos automáticos em identificar as mesmas e aplicar, adequadamente, o pulso desfibrilatório.

O analisador também possui uma saída de sinal de sincronismo para ser utilizada por cardioversores que demandam tal sinal de monitores cardíacos para a realização de descargas sincronizadas.

Os sinais de ECG e da saída para o osciloscópio são gerados a partir dos blocos de saída D/A do microcontrolador CY8C29666. Estes sinais são condicionados por blocos específicos, após serem gerados pelo microcontrolador, conforme mostrado na Figura 1. O sinal na saída para o osciloscópio apresenta a forma de onda do pulso desfibrilatório atenuada de 1.500 vezes.

Os sinais de ECG gerados, depois de condicionados, podem apresentar amplitudes que variam de $0,5 \mathrm{a}, 4,0 \mathrm{mV}$ pico a pico e taxas de batimento cardíaco que vão de 30 a 300 bpm. O sinal da saída de sincronismo é gerado pelo microcontrolador no instante em que o gerador de sinal de ECG gera a onda R, e tem amplitude de $5 \mathrm{~V}$ e largura de $100 \mathrm{~ms}$.

A interface com o usuário (teclado, display, buzzer, saída serial e impressora) é controlada pela placa processadora, que também é responsável pelo processamento do sinal captado e armazenamento das ondas amostradas.

Os circuitos do analisador estão divididos fisicamente em três placas distintas: placa atenuadora, que contém os circuitos atenuadores e os circuitos de proteção contra as altas tensões, placa processadora, constituída por um módulo CPU embarcado ("CPU embedded"), e placa base, que possui os circuitos de condicionamento e medição dos sinais, além de realizar a interface entre as placas e os periféricos.

\section{Procedimento para calibração das medições de energia}

Para a calibração das medições de energia, a incerteza padrão da medição foi determinada de acordo com a publicação da versão brasileira do EA-4/02 Expressão da Incerteza de Medição na Calibração (Instituto..., 1999) e do Joint Committee for Guides in Metrology - JCGM 100:2008 (Bureau..., 2008), seguindo o procedimento indicado pelo item 7 'Procedimento passo a passo para o cálculo da incerteza de medição' da versão brasileira do EA-4/02 (Instituto..., 1999).

O primeiro passo deste procedimento consiste em modelar a medição, de modo a expressar em termos matemáticos a dependência do mensurando (grandeza de saída) com as grandezas de entrada.

Considerando que a resistência de carga não apresenta indutância significativa, pode-se determinar o valor da energia aplicada por um pulso de descarga com duração finita e inferior a $T$ pela Equação 1:

$$
E=\frac{1}{R} \int_{0}^{T} V_{D E S C}^{2}(t) \cdot d t
$$

A tensão do pulso de descarga $V_{D E S C}$ é medida através dos canais $\mathrm{CH} 0$ e $\mathrm{CH} 1$ do conversor $\mathrm{A} / \mathrm{D}$ após ter sido atenuado no bloco atenuador e no bloco filtro de entrada (Figura 1). A atenuação teórica do bloco atenuador é de 3.007 vezes e as atenuações teóricas do bloco filtro de entrada para o canal $\mathrm{CH} 0$ e para o canal CH1 são iguais a 1,00 e 0,20 , respectivamente. As atenuações para os canais do conversor A/D são diferentes para que se possa calcular a energia do pulso desfibrilatório amostrado com melhor resolução (sem saturação) de modo a se obter melhor exatidão para a medida.

O modelo matemático para as medições de energia do desfibrilador desenvolvido é dado pela Equação 2:

$$
\begin{aligned}
& E_{\mathrm{CH} j}=\frac{1}{R} \int_{0}^{T} A T_{A T}^{2} \cdot A T_{\mathrm{CH} j}^{2} \cdot V_{\mathrm{CH} j}^{2}(t) \cdot d t= \\
& \frac{A T_{A T}^{2} \cdot A T_{\mathrm{CH} j}^{2}}{R} \cdot \int_{0}^{T} V_{\mathrm{CH} j}^{2}(t) \cdot d t, \quad j=0 \mathrm{ou} 1
\end{aligned}
$$


na qual: $[0, T]$ corresponde à janela de integração do analisador que apresenta uma largura igual a $24 \mathrm{~ms}$; $E_{\mathrm{CH} j}$ é a energia determinada a partir das medições realizadas pelo canal $\mathrm{CH} j, V_{\mathrm{CH} j}$ é a tensão na entrada do canal $\mathrm{CH} j, A T_{A T}$ é a atenuação do Bloco Atenuador e $A T_{\mathrm{CH} j}$ é a atenuação do Bloco Filtro de entrada para os canal $\mathrm{CH} j$, onde o canal $\mathrm{CH} j$ deve ser igual aos canais $\mathrm{CH} 0$ ou $\mathrm{CH} 1$ do conversor $\mathrm{A} / \mathrm{D}$.

$\mathrm{O}$ segundo passo deste procedimento relaciona todas as fontes de incerteza na forma de uma análise de incertezas. De acordo com a Equação 2, tem-se que as incertezas primárias relacionadas com as medições de energia através do canal $\mathrm{CH} 0\left(E_{\text {СН }}\right)$ são: $u(R), u\left(A T_{A T}\right), u\left(A T_{\mathrm{CH} O}\right)$ e $u\left(\int_{0}^{T} V_{\mathrm{CH} 0}^{2}(t) \cdot d t\right)$, e as incertezas primárias relacionadas com as medições de energia através do canal CH1 $\left(E_{\mathrm{CH} 1}\right)$ são: $u(R)$, $u\left(A T_{A T}\right), u\left(A T_{\mathrm{CH} 1}\right)$ e $u\left(\int_{0}^{T} V_{\mathrm{CH} 1}^{2}(t) \cdot d t\right)$.

A Tabela 1 apresenta a lista das fontes de incertezas relativas às medições da energia de um pulso desfibrilatório, apresentando as incertezas secundárias das quais derivam as incertezas primárias citadas.

No terceiro passo deste procedimento, a partir da Equação 2, e baseando-se nos métodos de cálculo da incerteza de medição determinados pela versão

Tabela 1. Principais fontes de incerteza para o método proposto.

Table 1. Main sources of uncertainty for the proposed method.

\begin{tabular}{|c|c|c|}
\hline Tipo & Símbolo & Descrição \\
\hline B & ---- & Condição ambiental (temperatura) \\
\hline A & $u(\bar{R})$ & Desvio padrão da média das medições da resistência de carga. \\
\hline $\mathrm{B}$ & $u(\nabla \bar{R})$ & Incerteza nas medições de resistência do multímetro (especificação do multímetro). \\
\hline & $u\left(\overline{V_{A T \mathrm{IN}}}\right)$ & Desvio padrão da média das medições da tensão na entrada do bloco atenuador. \\
\hline A & $u\left(\overline{V_{A T \text { OUT }}}\right)$ & Desvio padrão da média das medições da tensão de saída do bloco atenuador. \\
\hline B & $u\left(\Delta \overline{V_{A T \mathrm{IN}}}\right)$ & Incerteza nas medições da tensão de entrada do bloco atenuador. \\
\hline B & $u\left(\Delta \overline{V_{A T \mathrm{OUT}}}\right)$ & Incerteza nas medições da tensão de saída do bloco atenuador. \\
\hline A & $u\left(\overline{V_{F I L T-\mathrm{CH} 0 f i}}\right)$ & $\begin{array}{l}\text { Desvio padrão da média das medições da tensão na entrada do bloco filtro de entrada para um } \\
\text { sinal senoidal com frequência } f_{i} \text { e amplitude pico a pico igual a } 2,00 \mathrm{~V} \text {. }\end{array}$ \\
\hline $\mathrm{A}$ & $u\left(\overline{V_{F I L T-\mathrm{CH} 1 f i}}\right)$ & $\begin{array}{l}\text { Desvio padrão da média das medições da tensão na entrada do bloco filtro de entrada para um } \\
\text { sinal senoidal com frequência } f_{i} \text { e amplitude pico a pico igual a } 500 \mathrm{mV} \text {. }\end{array}$ \\
\hline $\mathrm{A}$ & $u\left(\overline{V_{\mathrm{CH} 0-f i}}\right)$ & $\begin{array}{l}\text { Desvio padrão da média das medições da tensão no canal } \mathrm{CH} 0 \text { para um sinal senoidal com } \\
\text { frequência } f_{i} \text { e amplitude pico a pico igual a } 2,00 \mathrm{~V} \text { aplicado na entrada do bloco filtro de entrada. }\end{array}$ \\
\hline $\mathrm{A}$ & $u\left(\overline{V_{\mathrm{CH} 1-f i}}\right)$ & $\begin{array}{l}\text { Desvio padrão da média das medições da tensão no canal } \mathrm{CH} 1 \text { para um sinal senoidal com } \\
\text { frequência } f_{i} \text { e amplitude pico a pico igual a } 500 \mathrm{mV} \text { aplicado na entrada do bloco filtro de entrada. }\end{array}$ \\
\hline B & $u\left(\Delta \overline{V_{F I L T-\mathrm{CHOfi}}}\right)$ & $\begin{array}{l}\text { Incerteza nas medições da tensão na entrada do bloco filtro de entrada para um sinal senoidal com } \\
\text { frequência } f_{i} \text { e amplitude pico a pico igual a } 2,00 \mathrm{~V} \text {. }\end{array}$ \\
\hline B & $u\left(\Delta \overline{V_{F I L T-\mathrm{CH} 1 f i}}\right)$ & $\begin{array}{l}\text { Incerteza nas medições da tensão na entrada do bloco filtro de entrada para um sinal senoidal com } \\
\text { frequência } f_{i} \text { e amplitude pico a pico igual a } 500 \mathrm{mV} \text {. }\end{array}$ \\
\hline B & $u\left(\Delta \overline{V_{\mathrm{CH} 0 f i}}\right)$ & $\begin{array}{l}\text { Incerteza nas medições da tensão no canal CH0 para um sinal senoidal com frequência } f_{i} \text { e } \\
\text { amplitude pico a pico igual a } 2,00 \mathrm{~V} \text { aplicado na entrada do bloco filtro de entrada. }\end{array}$ \\
\hline B & $u\left(\Delta \overline{V_{\mathrm{CH} 1 f i}}\right)$ & $\begin{array}{l}\text { Incerteza nas medições da tensão no canal CH1 para um sinal senoidal com frequência } f_{i} \mathrm{e} \\
\text { amplitude pico a pico igual a } 500 \mathrm{mV} \text { aplicado na entrada do bloco filtro de entrada. }\end{array}$ \\
\hline A & $u\left(\overline{E_{\mathrm{CH} 0-f i}}\right)$ & $\begin{array}{l}\text { Desvio padrão da média das medições de energia do analisador desenvolvido para um sinal no } \\
\text { canal } \mathrm{CH} 0 \text { do conversor gerado por um ciclo de onda senoidal com frequência } f_{i} \text { e amplitude pico } \\
\text { a pico igual a } 2,00 \mathrm{~V} \text { aplicado na entrada do bloco filtro de entrada. }\end{array}$ \\
\hline $\mathrm{A}$ & $u\left(\overline{E_{\mathrm{CH} 1-f i}}\right)$ & $\begin{array}{l}\text { Desvio padrão da média das medições de energia do analisador desenvolvido para um sinal no } \\
\text { canal } \mathrm{CH} 1 \text { do conversor gerado por um ciclo de onda senoidal com frequência } f_{i} \text { e amplitude pico } \\
\text { a pico igual a } 500 \mathrm{mV} \text { aplicado na entrada do bloco filtro de entrada. }\end{array}$ \\
\hline $\mathrm{A}$ & $u\left(\overline{T_{\mathrm{CH} j-f i}}\right)$ & $\begin{array}{l}\text { Desvio padrão da média das medições do período da tensão no canal } \mathrm{CH} j \text { do conversor } \mathrm{A} / \mathrm{D} \text { para } \\
\text { um sinal de frequência } f_{i} \text { aplicado na entrada do bloco filtro de entrada. }\end{array}$ \\
\hline B & $u\left(\Delta \overline{T_{\mathrm{CH} j-f i}}\right)$ & $\begin{array}{l}\text { Incerteza das medições do período da tensão no canal } \mathrm{CH} j \text { do conversor } \mathrm{A} / \mathrm{D} \text { para um sinal de } \\
\text { frequência } f_{i} \text { aplicado na entrada do bloco filtro de entrada. }\end{array}$ \\
\hline
\end{tabular}


brasileira do EA-4/02 (Instituto..., 1999) e pelo Guia para a Expressão da Incerteza de Medição (Bureau..., 2008), determina-se a Equação 3 para a variância combinada relativa para energias determinadas a partir das medições realizadas pelo canal $\mathrm{CH} j$ do conversor $\mathrm{A} / \mathrm{D}$, onde $\mathrm{CH} j$ é igual a $\mathrm{CH} 0$ ou $\mathrm{CH} 1$ :

$$
\begin{aligned}
& u_{r}^{2}\left(E_{\mathrm{CH} j}\right)=\left(\frac{u\left(E_{\mathrm{CH} j}\right)}{E_{\mathrm{CH} j}}\right)^{2}=\left(\frac{u(R)}{R}\right)^{2}+\left(\frac{u\left(A T_{A T}\right)}{A T_{A T}}\right)^{2}+ \\
& \left(\frac{u\left(A T_{\mathrm{CH} j}\right)}{A T_{\mathrm{CH} j}}\right)^{2}+\left(\frac{u\left(\int_{0}^{T} V_{\mathrm{CH} j}^{2} \cdot d t\right)}{\int_{0}^{T} V_{\mathrm{CH} j}^{2} \cdot d t}\right), j=0 \text { ou } 1
\end{aligned}
$$

As parcelas da Equação 3 são determinadas no quarto passo deste procedimento, que é dividido em quatro etapas que correspondem ao cálculo de cada parcela do lado direito da Equação 3.

Portanto, na primeira etapa deste passo, determinou-se a estimativa da resistência de carga $R$ e a sua variância combinada relativa. A resistência de carga $R$ foi determinada a partir da realização de 10 medições da resistência. As medições da resistência foram realizadas por um multímetro digital (Fluke, modelo 45), que apresenta, para medições na faixa de 0 a $300 \Omega$, uma resolução igual a $10 \mathrm{~m} \Omega$ e erro máximo declarado de $0,05 \%$ da leitura mais 2 dígitos mais $0,02 \Omega$. Através da repetição das medições, determinou-se a estimativa do valor da resistência de carga $\bar{R}$, e a incerteza Tipo A relacionada à resistência de carga $u(\bar{R})$.

A incerteza de Tipo $\mathrm{B}$, relacionada à incerteza nas medições do multímetro utilizado, $u(\nabla \bar{R})$, foi determinada a partir da variância associada com as especificações do multímetro para a medição da resistência de carga que foi calculada de acordo com as determinações do JCGM 100:2008, mais especificamente no seu item 4.3.7 (Bureau..., 2008).

A variância combinada de $R$ é dada pela Equação 4 .

$$
u^{2}(R)=u^{2}(\bar{R})+u^{2}(\nabla \bar{R})
$$

$\mathrm{Na}$ etapa seguinte do quarto passo deste procedimento, determinou-se a estimativa da atenuação do bloco atenuador e a sua variância combinada relativa. A atenuação do bloco atenuador foi determinada como sendo igual à relação entre o valor da tensão de entrada do bloco atenuador $V_{A T I N}$, pela tensão de saída do bloco atenuado $V_{A T \text { OUT }}$, como mostra o modelo matemático dado pela Equação 5, tendo sido obtida a partir da aplicação de uma tensão à entrada do bloco atenuador.

$$
A T_{A T}=\frac{V_{A T \mathrm{NN}}}{V_{A T \mathrm{OUT}}}
$$

As estimativas dos valores de $\overline{V_{A T I N}}$ e $\overline{V_{A T O U T}}$ foram obtidas a partir de 10 repetições das medições das tensões na entrada e saída do bloco atenuador. As fontes de incerteza Tipo A relacionadas à incerteza combinada da atenuação do bloco atenuador $u\left(A T_{A T}\right)$, são $u\left(\overline{V_{A T \mathrm{IN}}}\right)$ e $u\left(\overline{V_{A T O U T}}\right)$.

As fontes de incerteza Tipo B do bloco atenuador $u\left(\Delta \overline{V_{A T \mathrm{IN}}}\right)$ e $u\left(\Delta \overline{V_{A T \text { OUT }}}\right)$ foram calculadas de acordo com o item 4.3.7 do JCGM 100:2008 (Bureau..., 2008). Estas incertezas foram determinadas a partir das variâncias associadas com as especificações do multímetro para a medição de tensões.

As variâncias combinadas de $V_{A T \text { IN }}$ e $V_{A T \text { OUT }}$ são dadas pelas Equações 6 e 7:

$$
\begin{aligned}
& u^{2}\left(V_{A T \mathrm{IN}}\right)=u^{2}\left(\overline{V_{A T \mathrm{IN}}}\right)+u^{2}\left(\nabla \overline{V_{A T \mathrm{~N}}}\right) \\
& u^{2}\left(V_{A T \mathrm{OUT}}\right)=u^{2}\left(\overline{V_{A T \mathrm{OUT}}}\right)+u^{2}\left(\nabla \overline{V_{A T O U T}}\right)
\end{aligned}
$$

A variância combinada relativa da atenuação do bloco atenuador é dada pela Equação 8:

$\left(\frac{u\left(A T_{A T}\right)}{A T_{A T}}\right)^{2}=\left(\frac{u\left(V_{A T \mathrm{IN}}\right)}{V_{A T \mathrm{IN}}}\right)^{2}+\left(\frac{u\left(V_{A T \mathrm{OUT}}\right)}{V_{A T \mathrm{OUT}}}\right)^{2}$

$\mathrm{Na}$ terceira etapa deste quarto passo do procedimento, determinou-se a estimativa e a variância combinada relativa da atenuação do bloco filtro de entrada. A atenuação do bloco filtro de entrada foi determinada como sendo igual à média das relações entre a tensão de entrada e a tensão de saída do bloco filtro de entrada para sinais senoidais com frequência $f_{i}$ de $50,100,250$ e $500 \mathrm{~Hz}$.

O trabalho de Guedes e Moraes (2001) demonstra que o espectro de frequência de um pulso de $240 \mathrm{~J}$ gerado por um desfibrilador ECAFIX modelo DF-1, pulso este bastante similar aos gerados por outros equipamentos de descarga senoidal amortecida, não apresenta componentes significativas com frequências superiores a $500 \mathrm{~Hz}$. Os valores das frequências $f_{i}$ dos sinais aplicados foram definidos com base no trabalho Guedes e Moraes (2001).

A Equação 9 apresenta os modelos matemáticos dados para a atenuação do bloco filtro de entrada para uma tensão senoidal com frequência $f_{i}$ relativa aos canais $\mathrm{CH} 0$ e $\mathrm{CH} 1$ do conversor A/D.

$A T_{\mathrm{CH} j f i}=\frac{V_{F L L T-\mathrm{CH} j f i}}{V_{\mathrm{CH} j f i}} \quad j=0$ ou 1

As estimativas dos valores de $\overline{V_{F I L T-\mathrm{CH} 0 f i}}, \overline{V_{F L T-\mathrm{CH} 1 f i}}$, $\overline{V_{\mathrm{CH} 0 f i}}$ e $\overline{V_{\mathrm{CH} f i}}$ foram obtidas a partir de 10 repetições das medições das tensões na entrada e saída do bloco filtro de entrada.

As fontes de incerteza Tipo A relacionadas à incerteza combinada da atenuação do bloco filtro de entrada para o canal $\mathrm{CH} 0$ do conversor $\mathrm{A} / \mathrm{D}$ são 
$u\left(\overline{V_{F I L T-\mathrm{CH} 0 f i}}\right)$ e $u\left(\overline{V_{\mathrm{CH} 0 f i}}\right)$, e para o canal CH1 são $u\left(\overline{V_{F I L T-\mathrm{CH} 1 f i}}\right)$ e $u\left(\overline{V_{\mathrm{CH} l f i}}\right)$.

As fontes de incerteza Tipo $\mathrm{B}$ relacionadas à atenuação do bloco filtro de entrada são $u\left(\Delta \overline{V_{F I L T-C H 0 f i}}\right)$, $u\left(\Delta \overline{V_{F I L T-C H 1 f i}}\right), \quad u\left(\Delta \overline{V_{C H 0 f i}}\right)$ e $u\left(\Delta \overline{V_{C H 1 f i}}\right)$, e estão relacionadas com a incerteza do multímetro para a medição de tensões.

As variâncias combinada de $V_{F I L T-\mathrm{CH} j f i}$ e $V_{\mathrm{CH} j f i}$ são dadas pelas Equações 10 e 11.

$$
\begin{aligned}
& u^{2}\left(V_{F I L T-\mathrm{CH} j f i}\right)=u^{2}\left(\overline{V_{F I L T-\mathrm{CH} j f i}}\right)+u^{2}\left(\bar{\nabla} \overline{V_{F I L T-\mathrm{CH} j i}}\right) \\
& u^{2}\left(V_{\mathrm{CH} j i i}\right)=u^{2}\left(\overline{V_{\mathrm{CH} j f i}}\right)+u^{2}\left(\nabla \overline{V_{\mathrm{CH} j i}}\right)
\end{aligned}
$$

As variâncias combinadas relativas da atenuação do bloco filtro de entrada para uma frequência $f_{i}$, para os canais $\mathrm{CH} 0$ e $\mathrm{CH} 1$ do conversor A/D são dadas pela Equação 12.

$$
\begin{aligned}
& \left(\frac{u\left(A T_{\mathrm{CH} j f i}\right)}{A T_{\mathrm{CH} j i}}\right)^{2}=\left(\frac{u\left(V_{F I L T-\mathrm{CH} j i}\right)}{V_{F I L T-\mathrm{CH} j f i}}\right)^{2}+ \\
& \left(\frac{u\left(V_{\mathrm{CH} j i}\right)}{V_{\mathrm{CH} j i}}\right)^{2} \quad j=0 \text { ou } 1
\end{aligned}
$$

Como a atenuação do bloco filtro de entrada foi determinada como sendo a média aritmética da atenuação encontrada para cada uma das frequências $f_{i}$, a variância combinada da atenuação do bloco filtro de entrada é determinada pela Equação 13.

$$
u^{2}\left(A T_{\mathrm{CH} j}\right)=\frac{1}{16}\left[\begin{array}{l}
u^{2}\left(A T_{\mathrm{CH} j-50 \mathrm{~Hz}}\right)+u^{2}\left(A T_{\mathrm{CH} j-100 \mathrm{~Hz}}\right)+ \\
u^{2}\left(A T_{\mathrm{CH} j-250 \mathrm{~Hz}}\right)+u^{2}\left(A T_{\mathrm{CH} j-500 \mathrm{~Hz}}\right)
\end{array}\right]
$$

$\mathrm{Na}$ quarta e última etapa do quarto passo deste procedimento, determinou-se a estimativa e a variância combinada relativa de $\int V_{\mathrm{CH} 0}^{2}(t) \cdot d t$ e $\int V_{\mathrm{CH} 1}^{2}(t) \cdot d t$.

Para o cálculo da variância combinada relativa de $\int V_{\mathrm{CH} 0}^{2}(t) \cdot d t$ e $\int V_{\mathrm{CH} 1}^{2}(t) \cdot d t$ utilizou-se a relação existente entre o cálculo do valor eficaz da tensão de um sinal periódico e o valor da energia de um sinal (Maciel Junior, 2000).

A obtenção de $\int V_{i}^{2}(t) \cdot d t$ a partir do valor de energia $\left(E_{S}\right)$ e do valor eficaz da tensão de um sinal $V_{i}$ periódico em um intervalo de tempo igual a $T$, onde $T$ é um múltiplo inteiro do período de $V_{i}$, são apresentados nas Equações 14 e 15, respectivamente.

$$
\begin{aligned}
& E_{S}=\frac{1}{R} \int_{0}^{T} V_{i}^{2}(t) \cdot d t \rightarrow \int_{0}^{T} V_{i}^{2}(t) \cdot d t=E_{s} \cdot R \\
& V_{i \mathrm{RMS}}=\sqrt{\frac{1}{T} \int_{0}^{T} V_{i}^{2}(t) \cdot d t} \rightarrow \int_{0}^{T} V_{i}^{2}(t) \cdot d t=V_{i \mathrm{RMS}}^{2} \cdot T
\end{aligned}
$$

Para o levantamento do erro e da incerteza na medição da $\left(\int_{0}^{T} V_{\mathrm{CH} j}^{2}(t) \cdot d t\right)_{f i}$ aplicou-se, através do gerador de sinais, o mesmo sinal senoidal aplicado no procedimento realizado para o levantamento da atenuação do bloco filtro de entrada. Entretanto configurou-se este sinal para o modo burst, com a fase, o período e o retardo (delay) iguais a $0^{\circ}, 2$ e $0 \mathrm{~s}$, respectivamente.

O número de ciclos utilizado para os sinais é igual a $1,2,5$ e 10 ciclos, relativos aos sinais de $50,100,250$ e $500 \mathrm{~Hz}$, respectivamente.

A obtenção de $\overline{\left(\int_{0}^{T} V_{\mathrm{CH} j}^{2}(t) \cdot d t\right)_{f i}}$ a partir das medições de energia do analisador se faz pela Equação 16:

$$
\overline{\left(\int_{0}^{T} V_{\mathrm{CH} j}^{2}(t) \cdot d t\right)_{f i}}=\frac{R}{A T_{A T}^{2} \cdot A T_{\mathrm{CH} j}^{2}} \cdot \overline{E_{\mathrm{CH} j-f i}}, \quad j=0 \text { ou } 1
$$

Os valores de $R, A T_{A T}$ e $A T_{\mathrm{CH} j}$ são os obtidos durante o procedimento de calibração, sendo utilizados como parâmetro do software do analisador para o cálculo da energia. Consequentemente não adicionam erros para a obtenção do valor de $\overline{\left(\int_{0}^{T} V_{\mathrm{CH} j}^{2}(t) \cdot d t\right)_{f i}}$ a partir da energia medida pelo analisador.

Foram realizadas 10 repetições de medição da energia pelo analisador $E_{\mathrm{CH} j-f i}$, para cada um dos canais $\mathrm{CH} j$ e para cada uma das frequência $f_{i}$, para assim obter a estimativa do valor de energia $\overline{E_{\mathrm{CH} j-f i}}$ e a sua incerteza experimental $u\left(\overline{E_{\mathrm{CH} j-f i}}\right)$.

Como os valores de $R, A T_{A T}$ e $A T_{\mathrm{CH} j}$ não adicionam incertezas no cálculo de $\overline{\left(\int_{0}^{T} V_{\mathrm{CH} j}^{2}(t) \cdot d t\right)_{f i}}$, tem-se, de acordo com a versão brasileira do EA-4/02 (Instituto..., 1999) e do JCGM 100:2008 (Bureau..., 2008), que:

$$
u_{r}\left(\overline{\left(\int_{0}^{T} V_{\mathrm{CH} j}^{2}(t) \cdot d t\right)_{f i}}\right)=\frac{u\left(\overline{\left(\int_{0}^{T} V_{\mathrm{CH} j}^{2}(t) \cdot d t\right)_{f i}}\right)}{\left(\int_{0}^{T} V_{\mathrm{CH} j}^{2}(t) \cdot d t\right)_{f i}}=
$$

$\frac{u\left(\overline{E_{\mathrm{CH} j-f i}}\right)}{\overline{E_{\mathrm{CH} j-f i}}}, \quad j=0$ ou 1

O valor verdadeiro convencional (VVC) de $\int V_{\mathrm{CH} 0}^{2}(t) \cdot d t$ e $\int V_{\mathrm{CH} 1}^{2}(t) \cdot d t$. a ser adotado e o cálculo da incerteza do processo de calibração, foram determinados para avaliar a capacidade deste procedimento de calibração. Calculou-se $\left(\int_{0}^{T} V_{\mathrm{CH} j}^{2}(t) \cdot d t\right)_{V V C f i}$ a partir do valor eficaz da tensão no canal $\mathrm{CH} j$ do conversor $\mathrm{A} / \mathrm{D}$, medida através de um multímetro digital, no qual $\mathrm{CH}$ $j$ pode ser $\mathrm{CH} 0$ ou $\mathrm{CH} 1$, do período do sinal no canal $\mathrm{CH} j, V_{\mathrm{CH} j-f i}$, mensurado através de um osciloscópio digital, e do número de ciclos especificado para cada frequência $f_{i}$ do sinal, através da Equação 18:

$$
\left(\int_{0}^{T} V_{\mathrm{CH} j}^{2}(t) \cdot d t\right)_{V V C f i}=\overline{V_{\mathrm{CH} j} f i} \cdot N_{f i} \cdot \overline{T_{\mathrm{CH} j f i}}
$$


Considerando que não existe erro no número de ciclos a serem aplicados, tem-se que a incerteza definicional neste processo para $\left(\int_{0}^{T} V_{\mathrm{CH} j}^{2}(t) \cdot d t\right)_{V V C f i}$ é, portanto, determinada de acordo com a Equação 19:

$$
\begin{aligned}
& u_{r}^{2}\left(\left(\int_{0}^{T} V_{\mathrm{CH} j}^{2}(t) \cdot d t\right)_{V V C f i}\right)=\left(\frac{u\left(\int_{0}^{T} V_{\mathrm{CH} j}^{2}(t) \cdot d t\right)_{V V C f i}}{\left.\overline{\left(\int_{0}^{T} V_{\mathrm{CH} j}^{2}(t) \cdot d t\right)_{V V C f i}}\right)^{2}=}\right. \\
& \left(\frac{u\left(V_{\mathrm{CH} j f i}\right)}{\overline{V_{\mathrm{CH} j}}}\right)^{2}+\left(\frac{u\left(T_{\mathrm{CH} j f i}\right)}{\bar{T}_{\mathrm{CH} j i}}\right)^{2}
\end{aligned}
$$

As estimativas das incertezas relativas $u_{r}\left(\left(\int_{0}^{T} V_{\mathrm{CH} j}{ }^{2}(t) \cdot d t\right)_{V V C}\right)$ e $u_{r}\left(\overline{\left(\int_{0}^{T} V_{\mathrm{CH} j}^{2}(t) \cdot d t\right)}\right)$, e os erros relativos foram determinados como a média dos valores obtidos para cada frequência $f_{i}$.

No quinto passo deste procedimento, a partir da Equação 3 e dos resultados obtidos no quarto passo, calcula-se a variância combinada relativa para energias determinadas a partir das medições realizadas pelo canal $\mathrm{CH} j$ do conversor $\mathrm{A} / \mathrm{D}$, onde $\mathrm{CH} j$ é igual a $\mathrm{CH} 0$ ou CH1. Neste passo calcula-se também a incerteza relativa para as energias a partir das suas respectivas variâncias relativas.

O passo seguinte no procedimento constituiu-se da determinação das incertezas relativas expandidas $U_{r}\left(E_{\mathrm{CH} j}\right)=u_{r}\left(E_{\mathrm{CH} j}\right) \cdot k$ para uma probabilidade de abrangência de $95,45 \%$.

Como nenhuma das contribuições para a incerteza foi obtida de uma avaliação do Tipo A baseada em menos de 10 observações repetidas, de acordo com a publicação da versão brasileira do EA-4/02 (Instituto..., 1999), o critério de contabilidade é satisfeito, logo o fator da probabilidade de abrangência padronizado $k=2$ é utilizado.

O cálculo da incerteza relativa definicional, obtida no processo de calibração para a medição de energia do analisador, é realizado através da Equação 20.

$$
\begin{aligned}
& u r_{\text {calibraça o }}^{2}=\left(\frac{u(R)}{R}\right)^{2}+\left(\frac{u\left(A T_{A T}\right)}{A T_{A T}}\right)^{2}+\left(\frac{u\left(A T_{\mathrm{CH} j}\right)}{A T_{\mathrm{CH} j}}\right)^{2}+ \\
& \left(\frac{u\left(\int_{0}^{T} V_{\mathrm{CH} j}^{2} \cdot d t\right)_{V V C}}{\left(\int_{0}^{T} V_{\mathrm{CH} j}^{2} \cdot d t\right)_{V V C}}\right)^{2}, \quad j=0 \text { ou } 1
\end{aligned}
$$

A partir do valor da incerteza relativa definicional e da tolerância para o erro de medição de energia do analisador, determina-se o índice de capacidade $C_{m}$ do processo de calibração através da Equação 21:

$$
C_{m}=\frac{I T}{3 \cdot u r_{\text {calibração }}}
$$

na qual $I T$ corresponde à tolerância para o erro de medição de energia do analisador.

O índice de capacidade $C_{m}$ compara a variabilidade total permitida ao produto (ou tolerância de especificação) com a variação do processo (também chamada de tolerância natural). A capacidade de um sistema de medição pode ser utilizada para a avaliação do processo de medição.

\section{Resultados}

Durante a realização do procedimento a temperatura e a umidade relativa do ar foram registradas por um higrotermógrafo, que obteve como resultado temperaturas variando de 23 a $25^{\circ} \mathrm{C}$, e umidade relativa do ar variando de 70 a $76 \%$, sendo a incerteza do instrumento de medição igual a $1{ }^{\circ} \mathrm{C}$ para a temperatura e a $3 \%$ para a umidade relativa do ar.

A Tabela 2 apresenta os resultados obtidos para a estimativa e a variância da resistência de carga $R$, tensão na entrada do bloco atenuador $V_{A T \text { IN }}$ e tensão na saída do bloco atenuador $V_{A T \text { OUT }}$.

A Tabela 3 apresenta os resultados obtidos para a estimativa da atenuação do bloco atenuador $A T_{A T}$, calculados a partir dos resultados obtidos para a tensão de entrada $V_{A T \text { IN }}$, e tensão de saída do bloco atenuador $V_{A T \text { OUT }}$.

A Tabela 4 apresenta os resultados obtidos para a estimativa e variância da tensão de entrada do bloco filtro de entrada a partir de um sinal senoidal com 2,00 V de amplitude $V_{\text {FLLT-С } 0 \text { fi }}$, e com $500 \mathrm{mV}$ de amplitude $V_{\text {FLT-CH1 fi }}$, para frequências $f i$ iguais a $50,100,250$ e $500 \mathrm{~Hz}$ e as respectivas tensões de saída deste bloco, que são as tensões no canal $\mathrm{CH} 0, V_{\mathrm{CH} 0 f i}$, e no canal $\mathrm{CH} 1$ do conversor $\mathrm{A} / \mathrm{D}, V_{\mathrm{CH} 1 f i}$.

A Tabela 4 também apresenta os resultados obtidos das medições dos períodos $T_{\mathrm{CH} f i}$ e $T_{\mathrm{CH} 1 f f}$, dos sinais das tensões no canal $\mathrm{CH} 0$ e $\mathrm{CH} 1$ do conversor A/D, $V_{\text {Сно } f}$, e $V_{\text {СH } 1 f}$, respectivamente.

A Tabela 5 apresenta os resultados obtidos para as estimativas da atenuação do bloco filtro de entrada relativa ao canal $\mathrm{CH} 0$ do conversor $\mathrm{A} / \mathrm{D} A T_{\text {СH }}$, e relativa ao canal $\mathrm{CH} 1$ do conversor $\mathrm{A} / \mathrm{D} A T_{\mathrm{CH}}$, calculados a partir da média das estimativas e incertezas obtidas para a tensão de entrada e de saída do bloco filtro de entrada, respectivamente, para o canal CH0, $V_{\text {FLTTCH0 fi }}$, e $V_{\text {CH0 fi }}$, e para o canal CH1, $V_{\text {FLLTCH } 1 f i}$, e $V_{\text {CH1 fi. }}$.

A Tabela 6 apresenta os resultados obtidos para a estimativa e incertezas da integral do quadrado da tensão no canal do conversor $\mathrm{A} / \mathrm{D}$ obtidos a partir das medições de energia do analisador.

A Tabela 7 apresenta os resultados obtidos para a estimativa e incertezas da integral do quadrado da tensão no canal do conversor $\mathrm{A} / \mathrm{D}$ obtidos a partir do 
valor eficaz da tensão no canal do conversor $\mathrm{A} / \mathrm{D}$, do período do sinal e do número de ciclos.

A Tabela 8 apresenta os resultados para a incerteza expandida das medições de energia do analisador obtidos a partir da Equação 3 e dos resultados apresentados nas Tabelas 2, 3, 5 e 6 .

A Tabela 9 apresenta os resultados para a incerteza definicional na metodologia proposta obtidos a partir

Tabela 2. Estimativa e variância dos parâmetros $\mathrm{R}, \mathrm{V}_{\mathrm{AT} \text { IN }}, \mathrm{V}_{\mathrm{AT} \text { OUT}}$ * Table 2. Estimate and variance of $R, V_{A T I N}$ and $V_{A T O U T}$.

\begin{tabular}{ccccc}
\hline $\boldsymbol{q}$ & $\overline{\boldsymbol{q}}$ & $\boldsymbol{u}^{2}(\overline{\mathbf{q}})$ & $\boldsymbol{u}^{2}(\nabla \overline{\mathbf{q}})$ & $\boldsymbol{u}^{2}(\boldsymbol{q})$ \\
$\mathrm{R}$ & $50,14 \Omega$ & $2,100 \cdot 10^{-5} \Omega^{2}$ & $1,411 \cdot 10^{-3} \Omega^{2}$ & $1,432 \cdot 10^{-3} \Omega^{2}$ \\
$V_{\mathrm{AT}}$ & $49,91 \mathrm{~V}$ & $2,588 \cdot 10^{-4} \mathrm{~V}^{2}$ & $4,020 \cdot 10^{-3} \mathrm{~V}^{2}$ & $4,279 \cdot 10^{-3} \mathrm{~V}^{2}$ \\
$V_{\mathrm{ATOUT}}$ & $16,58 \mathrm{mV}$ & $1,179 \cdot 10^{-10} \mathrm{~V}^{2}$ & $5,910 \cdot 10^{-9} \mathrm{~V}^{2}$ & $6,028 \cdot 10^{-9} \mathrm{~V}^{2}$ \\
\hline
\end{tabular}

Tabela 3. Resultados relativos a atenuação do bloco atenuador.

Table 3. Results related to the attenuation of the attenuator block.

\begin{tabular}{|c|c|c|c|c|}
\hline$\overline{A T_{A T}}$ & $\left(\frac{u\left(V_{A T_{\mathrm{IN}}}\right)}{\overline{V_{A T_{\mathrm{IN}}}}}\right)$ & $\left(\frac{u\left(V_{A T_{\mathrm{OUT}}}\right)}{\overline{V_{A T_{\mathrm{OUT}}}}}\right)$ & $\left(\frac{u\left(\overline{A T_{A T}}\right)}{\overline{A T_{A T}}}\right)^{2}$ & $\left(\frac{u\left(\overline{A T_{A T}}\right)}{\overline{A T_{A T}}}\right.$ \\
\hline 3010 & $1,311 \cdot 10^{-3}$ & $4,684 \cdot 10^{-3}$ & $2,365 \cdot 10^{-5}$ & $4,863 \cdot 10^{-3}$ \\
\hline
\end{tabular}

Tabela 4. Resultados relativos a $V_{F I L T-\mathrm{CH} j i}, V_{\mathrm{CH} j-f i}$ e $T_{\mathrm{CH} j-f i}$ para CH jigual a CH0 e CH1 e figual a $50,100,250$ e $500 \mathrm{~Hz}$. Table 4. Results of $V_{F I L T-\mathrm{CH} j i}, V_{\mathrm{CH} j-f i}$ and $T_{\mathrm{CH} j-f i}$ to $\mathrm{CH} j$ equal to CHO and CHI and $f_{i}$ equal to 50, 100,250 and $500 \mathrm{~Hz}$.

\begin{tabular}{|c|c|c|c|c|c|c|}
\hline$q$ & $\begin{array}{c}\boldsymbol{C H} \\
\boldsymbol{j}\end{array}$ & $f_{i}(H z)$ & $\bar{q}$ & $u^{2}(\bar{q})$ & $\boldsymbol{u}^{2}(\nabla \overline{\boldsymbol{q}})$ & $u^{2}(q)$ \\
\hline \multirow{8}{*}{$V_{F I L T-\mathrm{CH} j f i}}$, & \multirow{4}{*}{$\mathrm{CHO}$} & 50 & 0,70121 & $2,6667 \cdot 10^{-12}$ & $1,7315 \cdot 10^{-7}$ & $1,7315 \cdot 10^{-7}$ \\
\hline & & 100 & 0,70134 & $5,0000 \cdot 10^{-12}$ & $1,7318 \cdot 10^{-7}$ & $1,7319 \cdot 10^{-7}$ \\
\hline & & 250 & 0,70144 & $2,6667 \cdot 10^{-12}$ & $1,7321 \cdot 10^{-7}$ & $1,7322 \cdot 10^{-7}$ \\
\hline & & 500 & 0,70149 & $2,3333 \cdot 10^{-12}$ & $1,7323 \cdot 10^{-7}$ & $1,7323 \cdot 10^{-7}$ \\
\hline & \multirow{4}{*}{$\mathrm{CH} 1$} & 50 & 0,17542 & $1,0000 \cdot 10^{-12}$ & $5,4743 \cdot 10^{-8}$ & $5,4344 \cdot 10^{-8}$ \\
\hline & & 100 & 0,17546 & $1,7778 \cdot 10^{-12}$ & $5,4750 \cdot 10^{-8}$ & $5,4752 \cdot 10^{-8}$ \\
\hline & & 250 & 0,17551 & $1,0000 \cdot 10^{-12}$ & $5,4758 \cdot 10^{-8}$ & $5,4759 \cdot 10^{-8}$ \\
\hline & & 500 & 0,17552 & $2,6667 \cdot 10^{-12}$ & $5,4760 \cdot 10^{-8}$ & $5,4763 \cdot 10^{-8}$ \\
\hline \multirow{8}{*}{$V_{\mathrm{CH} j-f i}$} & \multirow{4}{*}{$\mathrm{CHO}$} & 50 & 0,70125 & $4,4444 \cdot 10^{-12}$ & $1,7316 \cdot 10^{-7}$ & $1,7317 \cdot 10^{-7}$ \\
\hline & & 100 & 0,70119 & $3,2222 \cdot 10^{-12}$ & $1,7314 \cdot 10^{-7}$ & $1,7315 \cdot 10^{-7}$ \\
\hline & & 250 & 0,70033 & $3,2222 \cdot 10^{-12}$ & $1,7289 \cdot 10^{-7}$ & $1,7290 \cdot 10^{-7}$ \\
\hline & & 500 & 0,69730 & $6,2222 \cdot 10^{-12}$ & $1,7202 \cdot 10^{-7}$ & $1,7203 \cdot 10^{-7}$ \\
\hline & \multirow{4}{*}{$\mathrm{CH} 1$} & 50 & 0,87562 & $1,2889 \cdot 10^{-11}$ & $2,2708 \cdot 10^{-7}$ & $2,2709 \cdot 10^{-7}$ \\
\hline & & 100 & 0,87557 & $8,4444 \cdot 10^{-12}$ & $2,2706 \cdot 10^{-7}$ & $2,2707 \cdot 10^{-7}$ \\
\hline & & 250 & 0,87461 & $1,6000 \cdot 10^{-11}$ & $2,2674 \cdot 10^{-7}$ & $2,2676 \cdot 10^{-7}$ \\
\hline & & 500 & 0,87096 & $6,7778 \cdot 10^{-12}$ & $2,2554 \cdot 10^{-7}$ & $2,2555 \cdot 10^{-7}$ \\
\hline \multirow{8}{*}{$T_{\mathrm{CH} j-f i}$} & \multirow{4}{*}{$\mathrm{CHO}$} & 50 & 20,00 & $8,056 \cdot 10^{-5}$ & $3,536 \cdot 10^{-3}$ & $3,617 \cdot 10^{-3}$ \\
\hline & & 100 & 10,00 & $1,778 \cdot 10^{-5}$ & $5,741 \cdot 10^{-4}$ & $5,919 \cdot 10^{-4}$ \\
\hline & & 250 & 4,00 & $4,000 \cdot 10^{-6}$ & $1,415 \cdot 10^{-4}$ & $1,455 \cdot 10^{-4}$ \\
\hline & & 500 & 2,001 & $1,3611 \cdot 10^{-6}$ & $3,5368 \cdot 10^{-5}$ & $3,6729 \cdot 10^{-5}$ \\
\hline & \multirow{4}{*}{$\mathrm{CH} 1$} & 50 & 20,00 & $1,111 \cdot 10^{-4}$ & $3,536 \cdot 10^{-3}$ & $3,647 \cdot 10^{-3}$ \\
\hline & & 100 & 10,00 & $1,778 \cdot 10^{-5}$ & $5,741 \cdot 10^{-4}$ & $5,919 \cdot 10^{-4}$ \\
\hline & & 250 & 4,00 & $3,222 \cdot 10^{-6}$ & $1,415 \cdot 10^{-4}$ & $1,447 \cdot 10^{-4}$ \\
\hline & & 500 & 2,002 & $5,833 \cdot 10^{-7}$ & $3,537 \cdot 10^{-5}$ & $3,5953 \cdot 10^{-5}$ \\
\hline
\end{tabular}

Tabela 5. Resultados relativos à atenuação do bloco filtro de entrada para o canal $\mathrm{CH} 0$ do conversor A/D e para o canal CH1 do conversor $\mathrm{A} / \mathrm{D}$. Table 5. Attenuation of input filter block for channel $\mathrm{CHO}$ and channel $\mathrm{CH} 1$ of the A/D converter.

\begin{tabular}{|c|c|c|c|c|c|c|c|}
\hline $\mathrm{CH} j$ & $\overline{A T_{\mathrm{CH} j}}$ & $u^{2}\left(A T_{\mathbf{C H} j-50 \mathrm{~Hz}}\right)$ & $u^{2}\left(A T_{\mathrm{CH} j-100 \mathrm{~Hz}}\right)$ & $u^{2}\left(A T_{\mathrm{CH}_{j-250 \mathrm{z}}}\right)$ & $u^{2}\left(A T_{\mathbf{C H} j-500 \mathrm{~Hz}}\right)$ & $\left(\frac{u\left(A T_{\mathrm{CH} j}\right)}{\overline{A T_{\mathrm{CH} j}}}\right)^{2}$ & $\left(\frac{u\left(A T_{\mathrm{CH} j}\right)}{\overline{A T_{\mathrm{CH} j}}}\right)$ \\
\hline $\mathrm{CHO}$ & 1,0019 & $7,0422 \cdot 10^{-7}$ & $7,0457 \cdot 10^{-7}$ & $7,0682 \cdot 10^{-7}$ & $7,1434 \cdot 10^{-7}$ & $1,7619 \cdot 10^{-7}$ & $4,1975 \cdot 10^{-4}$ \\
\hline $\mathrm{CH} 1$ & 0,20073 & $8,2767 \cdot 10^{-8}$ & $8,3314 \cdot 10^{-8}$ & $8,3523 \cdot 10^{-8}$ & $8,4268 \cdot 10^{-8}$ & $5,1788 \cdot 10^{-7}$ & $7,1964 \cdot 10^{-4}$ \\
\hline
\end{tabular}


Tabela 6. Resultados relativos à $\left(\int_{0}^{T} V_{\mathrm{CH} j}^{2}(t) \cdot d t\right)_{f i}$ obtidos a partir das medições de energia realizadas pelo analisador desenvolvido.

Table 6. Results for $\left(\int_{0}^{T} V_{\mathrm{CH} j}^{2}(t) \cdot d t\right)_{f i}$ obtained from energy measurements performed by the analyzer developed.

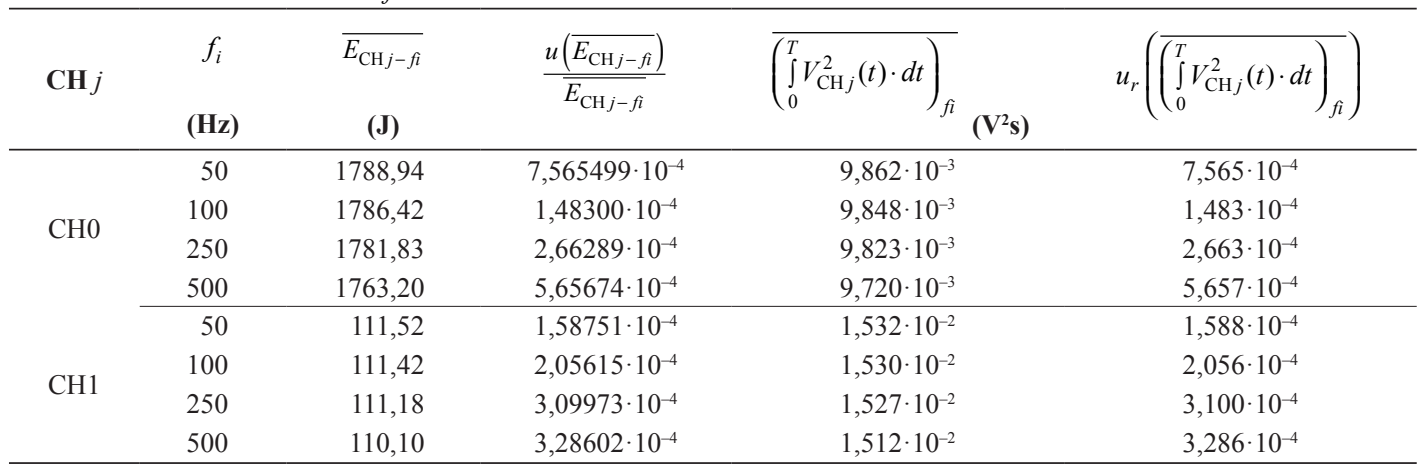

Tabela 7. Resultados relativos a $\left(\int_{0}^{T} V_{\mathrm{CH} j}^{2}(t) \cdot d t\right)_{V V C f i}$ obtidos a partir do valor eficaz da tensão no canal do conversor $\mathrm{A} / \mathrm{D}, V_{\mathrm{CH} j-f i}$, do período do sinal $T_{\mathrm{CH} j-f i}$, e do número de ciclos $N_{f i}$.

Table 7. Results for $\left(\int_{0}^{T} V_{\mathrm{CH} j}^{2}(t) \cdot d t\right)_{V V C f i}$ obtained from the RMS value of voltage in the channel of the A/D converter, $V_{\mathrm{CH} j-f i}$, the period of the signal $T_{\mathrm{CH}_{j-f i}}$, and the number of cycles $N_{f i}$.

\begin{tabular}{ccccc}
\hline $\mathbf{C H} j$ & $f_{i}(\mathbf{H z})$ & $N_{f i}$ & $\left(\int_{0}^{T} V_{\mathrm{CH} j}^{2}(t) \cdot d t\right)_{V V C f i}\left(\mathbf{V}^{2} \cdot \mathbf{s}\right)$ & $u_{r}\left(\nabla\left(\int_{0}^{T} V_{\mathrm{CH} j}^{2}(t) \cdot d t\right)_{f i}\right.$ \\
\hline \multirow{2}{*}{$\mathrm{CH} 0$} & 50 & 1 & $9,835 \cdot 10^{-3}$ & $3,066 \cdot 10^{-3}$ \\
& 100 & 2 & $9,833 \cdot 10^{-3}$ & $2,504 \cdot 10^{-3}$ \\
& 250 & 5 & $9,809 \cdot 10^{-3}$ & $3,072 \cdot 10^{-3}$ \\
& 500 & 10 & $9,729 \cdot 10^{-3}$ & $3,087 \cdot 10^{-3}$ \\
\hline \multirow{2}{*}{$\mathrm{CH} 1$} & 50 & 1 & $1,533 \cdot 10^{-2}$ & $3,068 \cdot 10^{-3}$ \\
& 100 & 5 & $1,533 \cdot 10^{-2}$ & $2,493 \cdot 10^{-3}$ \\
& 250 & 10 & $1,530 \cdot 10^{-2}$ & $3,055 \cdot 10^{-3}$ \\
& 500 & $1,518 \cdot 10^{-2}$ & $3,045 \cdot 10^{-3}$ \\
\hline
\end{tabular}

Tabela 8. Incerteza relativa expandida e erro relativo para a energia determinada a partir das medições do canal $\mathrm{CH} \mathrm{j}$ do conversor $\mathrm{A} / \mathrm{D}$, onde $\mathrm{CH}$ jé igual a $\mathrm{CH} 0$ ou $\mathrm{CH} 1$.

Table 8. Relative expanded uncertainty and error relative to the energy determined from measurements of the channel $\mathrm{CH} j$ of the A/D converter, where $\mathrm{CH}$ j is equal to $\mathrm{CHO}$ or $\mathrm{CH} 1$.

\begin{tabular}{cccc}
\hline $\begin{array}{c}\text { Incerteza } \\
\text { especificada } \\
\text { do analisador }\end{array}$ & $\mathbf{C H ~ j}$ & $\mathbf{U}_{\mathbf{r}}\left(\mathbf{E}_{\mathbf{C H} \mathbf{j}}\right)$ & $\mathbf{k}$ \\
\hline \multirow{2}{*}{$5 \%$} & $\mathrm{CH} 0\left(\right.$ para $\left.\mathrm{V}_{\mathrm{p}}<6.000 \mathrm{~V}\right)$ & $0,99 \%$ & 2 \\
& $\mathrm{CH} 1\left(\right.$ para $\left.\mathrm{V}_{\mathrm{p}}<1.000 \mathrm{~V}\right)$ & $1,00 \%$ & 2 \\
\hline
\end{tabular}

da Equação 20 e dos resultados apresentados nas Tabelas 2, 3, 5 e 7. A Tabela 9 apresenta também os resultados obtidos para o índice de capacidade $C_{m}$ do processo considerando uma incerteza máxima permitida para as medições de energia do analisador igual a 5\% e calculado conforme descrito em Oliveira (2010).

\section{Discussão}

Foiatto et al. (2009) propõem uma metodologia de calibração para analisadores de desfibriladores cardíacos a partir da integração de potência, utilizando um divisor de tensão e com base em medidas de intervalo de tempo e de sinais de tensão elétrica. Esta metodologia é realizada pelo método de transferência de valores, utilizando um desfibrilador para geração do pulso de energia, um divisor de tensão e um osciloscópio, que contenha no mínimo dois canais e funções matemáticas.

A metodologia apresenta como vantagens o fato de não necessitar conhecimento dos circuitos do analisador e de poder ser utilizada para a calibração de diferentes modelos de analisadores sem grandes alterações no seu procedimento. Como principal desvantagem tem-se a necessidade da utilização 
Tabela 9. Incerteza relativa definicional do procedimento de calibração proposto para a medição da energia determinada a partir das medições do canal $\mathrm{CH}$ j do conversor $\mathrm{A} / \mathrm{D}$, onde $\mathrm{CH}$ jé igual a $\mathrm{CH} 0$ ou $\mathrm{CH} 1$.

Table 9. Relative definitional uncertainty to the calibration procedure proposed for measuring the energy determined from measurements of channel $\mathrm{CH}$ j the $\mathrm{A} / \mathrm{D}$ converter, where $\mathrm{CH} j$ is equal to $\mathrm{CHO}$ or $\mathrm{CH}$.

\begin{tabular}{cccc}
\hline $\begin{array}{c}\text { Incerteza máxima } \\
\text { permitida para o } \\
\text { procedimento de } \\
\text { calibração }\end{array}$ & $\mathbf{C H ~ j ~}$ & $\mathbf{u}_{\text {r calibração }}$ & $\mathbf{C}_{\mathbf{m}}$ \\
\hline $1,67 \%$ & $\mathrm{CH} 0\left(\right.$ para $\left.\mathrm{V}_{\mathrm{p}}<6.000 \mathrm{~V}\right)$ & $0,57 \%$ & 2,92 \\
\hline
\end{tabular}

de um desfibrilador, que não é um equipamento tipicamente encontrado em laboratórios de calibração, e a exigência do osciloscópio utilizado apresentar funções matemáticas.

Por não estar dentre os seus objetivos, o trabalho de Foiatto et al. (2009) também não detalha o processo de obtenção da incerteza expandida da medição e nem o procedimento e os recursos necessários para que, a partir da forma de onda adquirida, se determine a energia da descarga, tais como as funções matemáticas que o osciloscópio deve possuir, o método de integração utilizado e o detalhamento do modelo matemático utilizado. Estas razões dificultam a adoção desta metodologia e, por contraposição, valorizam as características do presente estudo.

Vale salientar, portanto, que na metodologia apresentada utilizou-se de mais de um ponto de amostragem para destacar a importância de se considerar a resposta do equipamento analisado, tendo em vista o objetivo de auxiliar o leitor no desenvolvimento de metodologias de calibração.

A resposta em frequência do bloco filtro de entrada do analisador desenvolvido (Figura 1) apresenta o comportamento de um filtro passa-baixas com frequência de corte de $5 \mathrm{kHz}$. Observa-se, portanto, que o bloco filtro de entrada apresenta uma resposta praticamente constante para todo o espectro de frequência do pulso desfibrilatório de uma descarga senoidal amortecida. Este fato faz com que os resultados da atenuação do bloco filtro de entrada praticamente não se modifiquem quando a posição e o número de pontos de amostragem são alterados.

Outras formas de onda de pulso desfibrilatório apresentam um espectro de frequência diferente e mais largo que o espectro da descarga senoidal amortecida. Entretanto, mesmo para estas outras formas de onda, pode-se considerar que a frequência de corte apresentada pelo bloco filtro de entrada é suficientemente elevada para que não se necessite de mais de um ponto de amostragem.

A norma ANSI/AAMI DF80 (American..., 2003) é uma revisão conjunta das normas ANSI/ AAMI DF2 (American..., 1996) e ANSI/AAMI DF39, e não define o erro máximo admissível para os instrumentos de medição utilizados na realização dos ensaios por ela estabelecidos. Entretanto, a norma ANSI/AAMI DF2 (American..., 1996), a partir da qual ela foi baseada, estabelece que todos os instrumentos e equipamentos de ensaio utilizados na realização dos ensaios especificados por ela devem ter a maior exatidão possível, e não mais que um terço do erro máximo admissível da variável a ser medida ou $\pm 5 \%$ da variável a ser medida em que apenas limites máximos e mínimos são fornecidos.

Esta consideração está de acordo com a norma ISO 10012-1:1993, na qual o erro imputável à calibração deve ser tão pequeno quanto possível. $\mathrm{Na}$ maioria das áreas de medição não deveria ser maior do que um terço e, de preferência, um décimo do erro máximo admissível do equipamento comprovado quando em uso (Instituto..., 1993).

De acordo com as normas ANSI/AAMI DF80 (American..., 2003) e NBR IEC 60601-2-4 (Instituto..., 2005), a energia entregue não deve variar por mais que $\pm 15 \%$ da energia selecionada ou $\pm 3 \mathrm{~J}$, o maior valor dentre os dois. Portanto, tem-se que a incerteza máxima aceitável de um analisador para as medidas de energia é igual a $\pm 5 \%$ ou $\pm 1,0 \mathrm{~J}$, o que for maior dentre os dois.

A partir da calibração das medições de energia do analisador desenvolvido, constatou-se que este apresentou uma incerteza relativa expandida igual a 1,0\% para medições de energia a partir dos canais $\mathrm{CH} 0$ e $\mathrm{CH} 1$ do conversor $\mathrm{A} / \mathrm{D}$, conforme pode ser observado na Tabela 8. Estes resultados são adequados para um analisador de desfibriladores e cardioversores.

Uma avaliação da capacidade da metodologia utilizada para a calibração foi realizada. Nesta avaliação considerou-se o erro máximo admissível para as medições de energia do analisador de desfibriladores e cardioversores igual a $\pm 5 \%$ ou $\pm 1 \mathrm{~J}$, o que for maior dentre os dois. Para que o processo de calibração tenha uma probabilidade $\geq 99,73 \%$ de que o parâmetro mensurado realmente se encontre dentro das tolerâncias determinadas para o analisador, o índice de capacidade $C_{m}$ do processo de calibração deve ser $\geq 1$. Para tanto 
é necessário que o processo de calibração apresente um erro máximo não superior a $1,67 \%$ ou $0,33 \mathrm{~J}$.

Conforme pode ser observado na Tabela 9, o procedimento de calibração proposto apresentou uma incerteza de 0,57 e $0,58 \%$ para as medições realizadas através dos canais $\mathrm{CH} 0$ e $\mathrm{CH} 1$ do conversor $\mathrm{A} / \mathrm{D}$ respectivamente, e coeficientes de capacidade $C_{m}$ de 2,92 e 2,87 , respectivamente.

\section{Conclusão}

Através do estudo de caso do cálculo da incerteza da medição da energia apresentado neste estudo, apresenta-se os passos e as normas utilizados para a determinação de incertezas dos equipamentos. Deste modo, este artigo fornece a base necessária para o correto emprego de metodologias de calibração, contribuindo para o desenvolvimento de uma cultura metrológica.

\section{Referências}

American National Standards Institute - ANSI, Association for the Advancement of Medical Instrumentation - AAMI. ANSI/ AAMI DF2: Cardiac Defibrillator Devices. ANSI; 1996.

American National Standards Institute - ANSI, Association for the Advancement of Medical Instrumentation - AAMI. ANSI/AAMI DF80: Medical electrical equipment - Part 2-4: Particular requirements for the safety of cardiac defibrillators (including automated external defibrillators). ANSI; 2003.

Associação Brasileira de Normas Técnicas - ABNT. NBR ISO 10.012-1 Requisitos para garantia da qualidade para equipamento de medição. Rio de Janeiro: ABNT; 1993.
Associação Brasileira de Normas Técnicas - ABNT. NBR IEC 60601-1: Equipamento eletromédico - Parte 1 - Prescrições para segurança. Rio de Janeiro: ABNT; 1997.

Associação Brasileira de Normas Técnicas - ABNT. NBR IEC 60601-2-4: Equipamento eletromédico - Parte 2-4: Prescrições Particulares para Segurança de Desfibriladores Cardíacos. Rio de Janeiro: ABNT; 2005.

Bureau International des Poids et Mesures - BIPM. JCGM 100:2008 - GUM 1995 with minor corrections - Evaluation of measurement data - Guide to the expression of uncertainty in measurement. Paris: BIPM; 2008

Foiatto N, Pinto MVV, Hessel R. Calibração de analisadores de desfibriladores cardíacos. In: Semetro: Anais do $8^{\circ}$ Seminário Internacional de Metrologia Elétrica; 2009 jun. 17-19; João Pessoa, Brasil. João Pessoa, 2009. p. 1-4.

Guedes JR, Moraes R. Sistema para a análise de desfibriladores, cardioversores e esfigmomanômetros baseado em um microcomputador. Revista Brasileira de Engenharia Biomédica. 2001; 17(1):3-12.

Instituto Nacional de Metrologia, Qualidade e Tecnologia - INMETRO. Vocabulário Internacional de Metrologia: Conceitos fundamentais e gerais e termos associados (VIM 2008). Rio de Janeiro: INMETRO; 2009.

Instituto Nacional de Metrologia, Qualidade e Tecnologia - INMETRO. Versão Brasileira do EA-4/02 - Expressão da incerteza de medição na calibração. Rio de Janeiro: INMETRO; 1999.

Maciel Junior IS. Analisador de desfibrilador microcontrolado [dissertação]. João Pessoa: Universidade Federal de Paraiba; 2000 .

Oliveira CA. Analisador de desfibriladores e cardioversores [dissertação]. São Paulo: Escola Politécnica da Universidade de São Paulo. 2010.

\section{Autores}

